\title{
Optimal Implementations for Reliable Circadian Clocks
}

\author{
Yoshihiko Hasegawa,"* and Masanori Arita ${ }^{2,3}$ \\ ${ }^{1}$ Department of Biophysics and Biochemistry, Graduate School of Science, The University of Tokyo, Tokyo 113-0033, Japan \\ ${ }^{2}$ Center for Information Biology, National Institute of Genetics, Shizuoka 411-8540, Japan \\ ${ }^{3}$ RIKEN Center for Sustainable Resource Science, Kanagawa 230-0045, Japan
}

(Received 21 February 2014; revised manuscript received 19 May 2014; published 4 September 2014)

\begin{abstract}
Circadian rhythms are acquired through evolution to increase the chances for survival through synchronizing with the daylight cycle. Reliable synchronization is realized through two trade-off properties: regularity to keep time precisely, and entrainability to synchronize the internal time with daylight. We find by using a phase model with multiple inputs that achieving the maximal limit of regularity and entrainability entails many inherent features of the circadian mechanism. At the molecular level, we demonstrate the role sharing of two light inputs, phase advance and delay, as is well observed in mammals. At the behavioral level, the optimal phase-response curve inevitably contains a dead zone, a time during which light pulses neither advance nor delay the clock. We reproduce the results of phasecontrolling experiments entrained by two types of periodic light pulses. Our results indicate that circadian clocks are designed optimally for reliable clockwork through evolution.
\end{abstract}

PACS numbers: 87.18.Yt, 05.45.Xt, 87.18.Tt

Many terrestrial species, from cyanobacteria through to humans, adapt to sunlight and have acquired circadian oscillatory systems. Although their molecular implementation is species dependent [1], all exhibit a regular rhythm of $24 \mathrm{~h}$ that can be entrained by light input. Two fundamental properties are necessary for circadian clocks [2]: i.e., regularity to keep time precisely [3-5] and entrainability to adjust the internal time through light stimuli [6-8]. It is not easy to maximize entrainability and regularity simultaneously; higher entrainability implies more vulnerability to noise, whereas higher regularity signifies less flexibility to entrainment. We studied an optimal phase-response curve (PRC) problem for a oneinput pathway in [9]. In the present Letter, we generalize the calculations of [9] and formalize the optimal implementation problem for multiple input pathways without relying on specific oscillator models $[7,10,11]$. We show that the simultaneous maximization of entrainability and regularity entails several inherent properties of circadian clocks. At the molecular level, we rationalize the role sharing of multiple input pathways which was reported in murine circadian clocks [12,13]. At the behavioral level, we rationalize a time period during which the time is neither advanced nor delayed by light in an optimal clock [14]. Our theory can also explain different gene expression patterns when entrained by two different light pulses [15]. In this study we investigate the optimal implementations for multiple input pathways to achieve the maximal limit

Published by the American Physical Society under the terms of the Creative Commons Attribution 3.0 License. Further distribution of this work must maintain attribution to the author(s) and the published article's title, journal citation, and DOI. of entrainability and regularity. Although all circadian clocks transmit light signals through multiple pathways $[7,10,11]$, entrainment problems in multiple pathways have been little studied.

Circadian clocks keep time regularly under molecular noise [3-5]. It is also entrainable by periodic light stimuli. To consider these effects, we use an $N$-dimensional Langevin equation with respect to $x_{i}$, which is the concentration of the $i$ th molecular species: $\dot{x}_{i}=F_{i}(\boldsymbol{x} ; \rho)+$ $Q_{i}(\boldsymbol{x}) \xi_{i}(t)$, where $F_{i}(\boldsymbol{x} ; \rho)$ is the $i$ th reaction rate $(i=1,2, \ldots, N), Q_{i}(\boldsymbol{x})$ is a multiplicative noise term, and $\xi_{i}(t)$ is white Gaussian noise with $\left\langle\xi_{i}(t) \xi_{j}\left(t^{\prime}\right)\right\rangle=$ $2 \delta_{i j} \delta\left(t-t^{\prime}\right)$. To incorporate the effect of light, we introduce a light-sensitive parameter $\rho$ which is perturbed as $\rho \rightarrow \rho+d \rho$ when stimulated by light. We quantify regularity by the temporal variance in the oscillation. From [9], the period variance is known to be $\mathcal{V}_{T} \simeq T^{3}\left(4 \pi^{3}\right)^{-1} \int_{0}^{2 \pi} \sum_{i=1}^{N} U_{i}(\theta)^{2} Q_{i}(\theta)^{2} d \theta$, where $T$ is the period of the unperturbed oscillator, $\boldsymbol{U}(\phi)=$ $\left(U_{1}(\phi), \ldots, U_{N}(\phi)\right)$ is the infinitesimal PRC (iPRC) defined by $\boldsymbol{U}(\phi)=\left.\nabla_{x} \phi\right|_{x=x_{\mathrm{LC}}(\phi)}$, and $\boldsymbol{x}_{\mathrm{LC}}(\phi)$ is a point on the limit-cycle trajectory at phase $\phi \in[0,2 \pi)$. Entrainability is quantified as the width of the Arnold tongue. The phase dynamics with periodic input signals are described by a tilted periodic potential. If stable points exist in the potential, the oscillator can be entrained by input signals [16]. Thus we can discuss entrainability without considering noise because the existence of stable points does not depend on noise. Therefore, we set $Q_{i}(\boldsymbol{x})=0$. Let $p(\omega t)$ be an input signal with angular frequency $\omega$. For a weak input signal $d \rho=\chi p(\omega t)(\chi \geq 0$ is the signal strength), we obtain $\dot{\phi}=\Omega+\chi Z(\phi) p(\omega t)(\phi \in[0,2 \pi)$ is the phase and $\Omega=2 \pi / T)$, where 


$$
Z(\phi)=\sum_{i=1}^{N} Z_{i}(\phi), \quad Z_{i}(\phi)=\frac{\partial F_{i}(\phi ; \rho)}{\partial \rho} U_{i}(\phi),
$$

with $F_{i}(\phi ; \rho)=F_{i}\left(x_{\mathrm{LC}}(\phi) ; \rho\right) . \quad Z_{i}(\phi)$ quantifies phase shift due to the perturbation of $\rho$ in the $i$ th coordinate. We hereafter refer to $Z(\phi)$ as the parametric PRC (pPRC) $[9,17]$. The dynamics of a slow variable defined by $\psi=\phi-\omega t$ obeys

$$
\dot{\psi}=\Omega-\omega+\chi \Theta(\psi),
$$

where $\Theta(\psi)=(2 \pi)^{-1} \int_{0}^{2 \pi} Z(\psi+\theta) p(\theta) d \theta$. The width of the Arnold tongue is given by $\chi \mathcal{E}$, where $\mathcal{E}=(2 \pi)^{-1} \int_{0}^{2 \pi} Z(\theta)$ $\left\{p\left(\theta-\psi_{M}\right)-p\left(\theta-\psi_{m}\right)\right\} d \theta$ with $\psi_{M}=\operatorname{argmax}_{\psi} \Theta(\psi)$ and $\psi_{m}=\operatorname{argmin}_{\psi} \Theta(\psi)$ [9,18]. Optimal circadian clocks are derived as optimal PRCs $U_{i}(\phi)$ which were obtained with the variational method by maximizing the entrainability $\mathcal{E}$ subject to constant variance $\mathcal{V}_{T}=\sigma_{T}^{2}: U_{i}(\phi)=$ $\pi^{2} T^{-3} \lambda^{-1} Q_{i}(\phi)^{-2}\left\{p\left(\phi-\psi_{M}\right)-p\left(\phi-\psi_{m}\right)\right\} \partial_{\rho} F_{i}(\phi ; \rho)$ ( $\lambda$ is a Lagrange multiplier [19]) [9].

We generalize the above scheme to multiple input signals. Suppose there are $M$ input pathways and parameters $\rho_{1}, \rho_{2}, \ldots, \rho_{M}$ are affected as $\rho_{i} \rightarrow \rho_{i}+d \rho_{i}$ under light stimuli. By introducing an auxiliary scaling parameter $s_{i}$ defined by $d \rho_{i}=s_{i} d \rho$, each parameter perturbation is reparametrized as

$$
\rho_{i} \rightarrow \rho_{i}+d \rho_{i} \Rightarrow \tilde{\rho}_{i}+s_{i} \rho \rightarrow \tilde{\rho}_{i}+s_{i}(\rho+d \rho),
$$

where $\tilde{\rho}_{i}=\rho_{i}-s_{i} \rho$. Through this reparametrization, the multiple perturbations can be reduced to a single parameter case with respect to $\rho$. In this instance, $\rho$ in Eq. (3) is a hypothetical parameter and has no correspondence to actual reaction rates.

Light stimuli generally affect the rate constants, but the detailed mechanisms vary between organisms [21]. Our model can cover these different entrainment mechanisms. We first explain a simple case [Fig. 1(a)], and then generalize it. In Fig. 1(a), we assume that light stimuli enhance the synthesis rate of $x_{2}$, where the dynamics of $x_{2}$
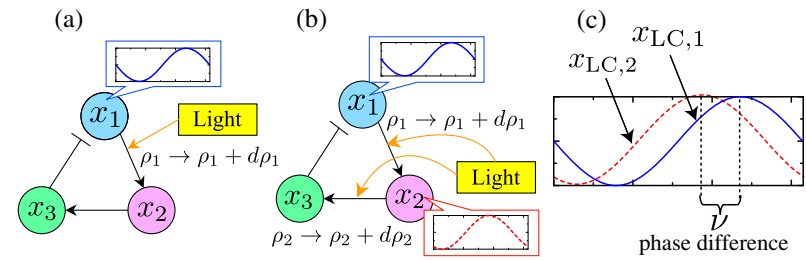

FIG. 1 (color online). Examples of (a) one-light input pathway and (b) two-light input pathway cases. The insets describe the time course of molecular species that are affected by light stimuli. In (b) the light-sensitive molecular species correspond to $x_{1}$ and $x_{2}$, which exhibit peaks at different phase (this difference is given by $\nu$ ). (c) The phase difference $\nu$ is defined by the difference between the $k_{1}$ th and $k_{2}$ th molecular species. can be described by $\dot{x}_{2}=\rho_{\text {syn }} x_{1}+\rho_{\text {deg }} x_{2}$ where $\rho_{\text {syn }}>0$ and $\rho_{\mathrm{deg}}<0$ are the synthesis and degradation rates, respectively. Because the synthesis rate increases when stimulated by light, by taking $\rho=\rho_{\text {syn }}$ ( $\rho$ is the lightsensitive parameter), the rate equation can be divided into $\rho$-dependent and $\rho$-independent parts $\dot{x}_{2}=F_{2}(\boldsymbol{x} ; \rho)=$ $\rho_{\text {deg }} x_{2}+\rho x_{1}=\tilde{F}_{2}(\boldsymbol{x})+\rho x_{1}$, where $\tilde{F}_{2}(\boldsymbol{x})$ denotes terms not including $\rho$ in $F_{2}(x ; \rho)$. We next consider a generic case with two-input pathways $(M=2)$. Let us assume that light stimuli affect parameters $\rho_{1}$ and $\rho_{2}$ (e.g., translation, transcription, or degradation rate) which depend on $k_{1}$ th and $k_{2}$ th molecular species and affect $j_{1}$ th and $j_{2}$ th species, respectively [e.g., $k_{1}=1, k_{2}=2, j_{1}=2$, and $j_{2}=3$ in Fig. 1(b)]. The rate equations $F_{j_{i}}(\boldsymbol{x} ; \rho)$ of $j_{1}$ th and $j_{2}$ th are described as

$$
\dot{x}_{j_{i}}=\tilde{F}_{j_{i}}(\boldsymbol{x})+\rho_{i} x_{k_{i}}=\underbrace{\tilde{F}_{j_{i}}(\boldsymbol{x})+\tilde{\rho}_{i} x_{k_{i}}}_{\rho \text {-independent }}+\underbrace{s_{i} \rho x_{k_{i}}}_{\rho \text {-dependent }},
$$

where $i=1,2$ and $\tilde{F}_{j_{i}}(\boldsymbol{x})$ denotes terms not including $\rho_{i}$ in $F_{j_{i}}(\boldsymbol{x} ; \rho)$. From Eq. (1), we see that PRCs are only concerned with the derivative of $F_{j_{i}}(\boldsymbol{x} ; \rho)$ with respect to $\rho$, which is specifically given by $\partial_{\rho} F_{j_{i}}(\boldsymbol{x} ; \rho)=s_{i} x_{k_{i}}$. Consequently, the $\rho$-independent part in Eq. (4) plays no role in the formation of optimal PRCs. We approximate $x_{k}$ by the $k$ th coordinate of $\boldsymbol{x}_{\mathrm{LC}}$, denoted as $x_{\mathrm{LC}, k}(\phi)$ in Eq. (4). Because $x_{\mathrm{LC}, k}(\phi)$ oscillates, we model it with a sine curve. the $k_{1}$ th and $k_{2}$ th molecular species may peak at different times as shown in Fig. 1(b), where the time courses of the $k_{1}$ th and $k_{2}$ th molecular species is described in the insets by solid and dashed lines, respectively. Therefore, the time course is approximated by $x_{\mathrm{LC}, k_{i}}(\phi)=1-\alpha_{i} \sin \left(\phi+u_{k_{i}}\right)$ for $i=1,2$, where $u_{k_{1}}$ and $u_{k_{2}}$ are the initial phases of the $k_{1}$ th and $k_{2}$ th molecular species, the amplitude $\alpha_{i}$ being assumed as identical $\alpha_{1}=\alpha_{2}=\alpha(0 \leq \alpha \leq 1)$. Let $\nu$ be the phase difference between the $k_{1}$ th and $k_{2}$ th molecular species, i.e., $\nu=u_{k_{2}}-u_{k_{1}}$ [Fig. 1(c)]. This parameter plays an important role in optimization.

Circadian clocks are entrained by sunlight whose intensity is determined by $24 \mathrm{~h}$ periodic solar irradiance. The solar irradiance $I$ with respect to the zenith angle $\vartheta$ is given by $I=I_{0} \cos \vartheta$, where $I_{0}$ is the irradiance at $\vartheta=0$, and $I$ vanishes when the sun is below the horizon [22]. Thus, we approximate its time course by $p(\omega t)=\sin (\omega t)$ for $0 \leq \bmod (\omega t, 2 \pi)<\pi$ (day) and $p(\omega t)=0$ for $\pi \leq$ $\bmod (\omega t, 2 \pi)<2 \pi \quad$ (night) with $\bmod (x, y)=x \bmod y$, which we call solar radiation input [9].

Our model starts from the two-input case $(M=2)$. The noise term was introduced only for input molecules $\left[Q_{j_{1}}(\phi)=\sqrt{q}, Q_{j_{2}}(\phi)=\sqrt{q}\right.$ and $Q_{i}(\phi)=0$ otherwise, where $q$ is the noise intensity], and we set $u_{k_{1}}=0$ (i.e., $\nu=u_{k_{2}}$ ), $T=1, \sigma_{T}^{2}=1$, and $q=1$ without loss of generality. We then need to specify additional parameters $s_{1}$ and $s_{2}$ [Eq. (3)] that determine the strength of the input 
signal relative to $\rho$. If we are concerned with pPRCs (experimentally observed PRCs) only, the sign of $s_{i}$ (positive or negative) does not play any role, because $s_{i}$ is squared in the optimal pPRCs. We set $s_{1}=s_{2}=1$ for simplicity; this corresponds to assuming equal weight for each light input pathway.

Figure 2(a) shows the $\nu$ dependence of maximal entrainability for $\alpha=0.5$ (solid line) and $\alpha=1$ (dashed line). For $\alpha=0.5$, the maximum was achieved at $\nu=0$, where no phase difference existed. However, upon increasing $\alpha$ to 1, maximal entrainability was achieved at two points, $\nu=0$ and $\nu=1.47$ [Figs. 3(a) and 3(b)]. Interestingly, optimality can be attained in the presence of phase difference. From Eq. (1), we divide the pPRC $Z(\phi)$ into contributions from two input pathways $Z(\phi)=Z_{j_{1}}(\phi)+Z_{j_{2}}(\phi)$, where $Z_{j_{1}}(\phi)$ and $Z_{j_{2}}(\phi)$ quantify the phase shift produced by the 1st and 2nd input pathways, respectively. Optimal $Z(\phi), Z_{j_{1}}(\phi)$, and $Z_{j_{2}}(\phi)$ for the two-input case are plotted in Figs. 3(a) and 3(b). Figures 3(c) and 3(d) are the corresponding time course of the $k_{1}$ th and $k_{2}$ th molecular species concentration. We see that optimal PRCs $Z(\phi)$ in Figs. 3(a) and 3(b) are very similar to experimentally observed PRCs in which there is a dead zone, a time during which light neither advanced nor delayed the clock $[1 \lesssim \phi \lesssim 2$ in Figs. 3(a) and 3(b)]. Intriguingly, experimental studies in different species reported the existence of the dead zone [14]. Although cases with $\nu=0$ and $\nu=1.47$ achieved the same entrainability, $Z(\phi)$ for $\nu=0$ is asymmetric with respect to horizontal axis, which entails an asymmetric Arnold tongue. Thus for a symmetric Arnold tongue, only $\nu=1.47$ can achieve maximal entrainability. We calculated the $\nu$ dependence of the dead zone length $L$ (length of null parts in PRCs [19]) in Fig. 2(b) for $\alpha=0.5$ (solid line) and $\alpha=1.0$ (dashed line). In Fig. 2(b), $L$ quickly diminishes around $\nu=\pi$, showing that a dead zone always appears in optimal PRCs except for a singular point $\nu=\pi$.

The fundamental difference of $\nu=1.47$ from $\nu=0$ is the role sharing of two PRCs $Z_{j_{1}}$ and $Z_{j_{2}}$ [Fig. 3(b)]. $Z_{j_{1}}$ is responsible for the phase advance and $Z_{j_{2}}$ for the delay

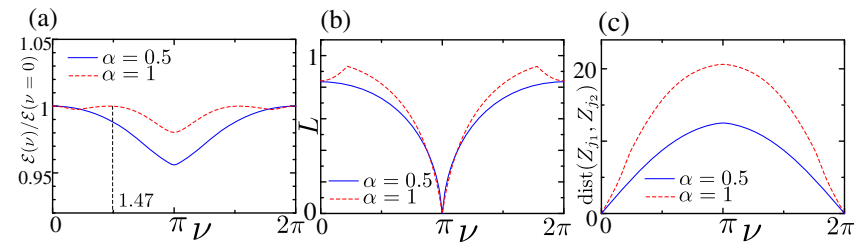

FIG. 2 (color online). (a) Normalized entrainability as a function of $\nu$ for $\alpha=0.5$ (solid line) and $\alpha=1$ (dashed line). The value is normalized by the entrainability at $\nu=0$ [i.e., $\mathcal{E}(\nu) / \mathcal{E}(\nu=0)]$. For $\alpha=1$, maximal is achieved at $\nu=0$ and 1.47. (b) Dead zone length $L$ as a function of the phase difference $\nu$ for $\alpha=0.5$ (solid line) and $\alpha=1$ (dashed line). (c) Distance between $Z_{j_{1}}(\phi)$ and $Z_{j_{2}}(\phi)$ as a function of $\nu$ for $\alpha=0.5$ (solid line) and $\alpha=1$ (dashed line). (the positive part of $Z_{j_{1}}$ is larger than the negative part and vice versa). This effect was observed for all $\nu$ values except $\nu=0$, as shown below. We quantify the distance between $Z_{j_{1}}$ and $Z_{j_{2}}$ by $\operatorname{dist}\left(Z_{j_{1}}, Z_{j_{2}}\right)=$ $\sqrt{\int_{0}^{2 \pi}\left\{Z_{j_{1}}(\theta)-Z_{j_{2}}(\theta)\right\}^{2} d \theta}$, which becomes larger when the two PRCs play more compensatory roles. The distance calculated as a function of $\nu$ for $\alpha=0.5$ (solid line) and $\alpha=1$ (dashed line) is shown in Fig. 2(c) where the distance is maximal exactly at $\nu=\pi$. When a phase difference $(\nu>0)$ exists, this role sharing between two-input pathways always yields a synchronization advantage. There is experimental evidence for advance and delay roles of Per 1 and Per 2 , respectively, in mice [12]. In this regard, Ref. [13] observed a period dependence of Per 1 and Per 2 knockout mutants on the intensity of constant light. We can reproduce this result with optimal PRCs as follows. Note that entrainability is maximal at $\nu=1.47$ for $\alpha=1$ [Fig. 3(b)]. Consequently, we set the clock parameters to $\alpha=1$ and $\nu=1.47$. Under a constant light condition, the input signal is modeled by $d \rho=\chi p(t)$, where $p(t)=1$. By integrating the phase equation $\dot{\phi}=\Omega+\chi Z(\phi) p(t)$ from $t=0$ to $t=T$, where $\phi(t=0)=0$, the phase at time $T$ with input strength $\chi$ is given by $\phi(T ; \chi)=$ $2 \pi+(2 \pi)^{-1} T \chi \int_{0}^{2 \pi} Z(\theta) d \theta$. For weak $\chi$, the period $T_{\chi}$, which is the period under a constant light condition, is approximated by $T_{\chi} / T \simeq \phi(T ; 0) / \phi(T ; \chi) \simeq 1-T \chi\left(4 \pi^{2}\right)^{-1}$ $\int_{0}^{2 \pi} Z(\theta) d \theta[17,19]$. Assuming $x_{j_{1}}=[$ Per 1$]$ and $x_{j_{2}}=$ [Per2], we simulated Per 1 and Per2 mutants by setting

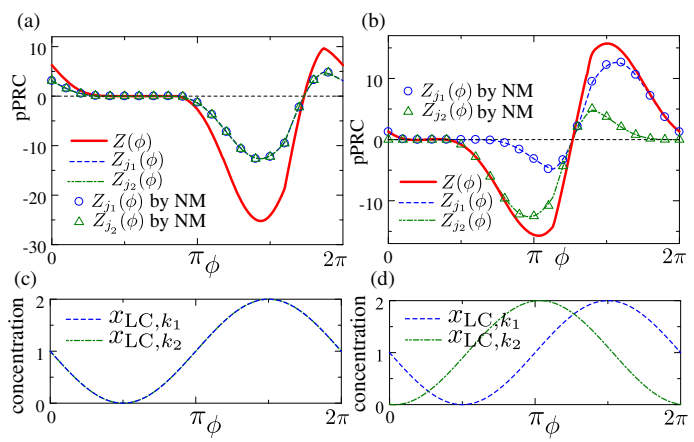

FIG. 3 (color online). (a)-(b) Optimal PRCs $\left[Z(\phi), Z_{j_{1}}(\phi)\right.$, and $\left.Z_{j_{2}}(\phi)\right]$ for (a) $\alpha=1$ and $\nu=0$ and (b) $\alpha=1$ and $\nu=1.47$. In (a) and (b), $Z(\phi), Z_{j_{1}}(\phi)$, and $Z_{j_{2}}(\phi)$ obtained with the variational method, are plotted with thick solid lines, dashed lines, and dot-dash lines, respectively. $Z_{j_{1}}(\phi)$ and $Z_{j_{2}}(\phi)$ obtained with a numerical method (NM) are plotted with circles and triangles, respectively [19]. In (a), PRCs that are symmetric with respect to the horizontal axis or $\phi=3 \pi / 2$ are also optimal. In (b), PRCs that are symmetric with respect to the horizontal axis are also optimal. (c)-(d) Time course of the molecular species concentration for (c) $\alpha=1$ and $\nu=0$ and (d) $\alpha=1$ and $\nu=1.47$. Two species $x_{\mathrm{LC}, k_{1}}$ and $x_{\mathrm{LC}, k_{2}}$ are plotted with dashed and dot-dash lines. In (a) and (c), dashed and dot-dashed lines are indistinguishable. 
$Z(\phi)=Z_{j_{2}}(\phi)$ and $Z(\phi)=Z_{j_{1}}(\phi)$, respectively. When increasing the intensity $\chi$ of constant light, the period ratio $T_{\chi} / T$ increases for Per 1 mutant and decreases for Per 2 mutant. This result agrees with the experimental evidence (Fig. 2 in [13]).

Our model can further suggest insights into the molecular mechanism of the clock. In hamsters, Schwartz et al. [15] reported different gene expressions of Per 1 and Per2 when entrained by two types of periodic light pulses that have short $(23.33 \mathrm{~h})$ and long $(24.67 \mathrm{~h})$ periods. Let us reproduce Schwartz's experiment in our optimization framework with two inputs. We again set $\alpha=1$ and $\nu=1.47$ and assume $x_{j_{1}}=[$ Per 1$]$ and $x_{j_{2}}=[$ Per 2$]$. Reference [15] applied a periodic light pulse of $1 \mathrm{~h}$ duration, which we modeled with a periodic $\delta$ function,

$$
p(\omega t)=2 \pi \delta(\bmod (\omega t, 2 \pi))
$$

where a factor $2 \pi$ ensures $\Theta(\psi)=(2 \pi)^{-1} \int_{0}^{2 \pi} p(\theta-\psi)$ $Z(\theta) d \theta=Z(\psi)$. Given the periodic light pulse [Eq. (5)], the entrainment phase $\psi_{\text {st }}$ (i.e., the circadian time at which hamsters receive the light pulses) can be determined by

$$
\Omega-\omega+\chi \Theta\left(\psi_{\mathrm{st}}\right)=0, \Theta^{\prime}\left(\psi_{\mathrm{st}}\right)<0,
$$

where we used Eq. (2). Thus, $\psi_{\text {st }}$ can be given as a solution of $Z\left(\psi_{\mathrm{st}}\right)=(\omega-\Omega) / \chi$ with $Z^{\prime}\left(\psi_{\mathrm{st}}\right)<0$. For the long $(\omega<\Omega)$ and short $(\omega>\Omega)$ pulses, $(\omega-\Omega) / \chi$ becomes negative and positive, respectively. This shows that the long and short pulses always act on hamsters at early $[\phi=1.3-3.2$; purple in Figs. 4(a) and 4(b)] and late $[\phi=4.7-0.47$; orange in Figs. 4(a) and 4(b)] subjective night, respectively. The effects of the light pulse on the circadian clock depend on the concentration of $x_{k_{1}}$ and $x_{k_{2}}$
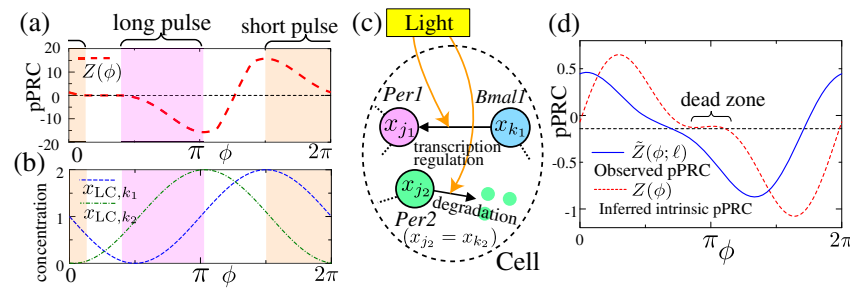

FIG. 4 (color online). (a)-(c) Theoretical reproduction of a light entrainment experiment involving hamsters [15]. (a) Optimal pPRC $Z(\phi)$ of the two-input case $M=2$ with $\alpha=1$ and $\nu=1.47$ [Fig. 3(b)]. (b) The time course of the molecular species concentration of $x_{\mathrm{LC}, k_{1}}$ (dashed lines) and $x_{\mathrm{LC}, k_{2}}$ (dotdash lines) for $\alpha=1$ and $\nu=1.47$. (c) Molecular implementation of the murine light entrainment mechanism. The model can be described by Eq. (4) with $x_{j_{1}}=[\operatorname{Per} 1], x_{k_{1}}=[$ Bmal1], and $x_{j_{2}}=x_{k_{2}}=[$ Per 2$]$. (d) Observed (solid line) and inferredintrinsic $\left(N_{\mu}=2, \chi=1\right.$; dashed line) pPRCs of humans as a function of the onset of pulses. Observed pPRC is brought from Ref. [24], which measured pPRC with $\ell=6.7$ h light pulses. The horizontal dashed line indicates anticipated phase delay [24]. at these phases [Eq. (4)]. For the long pulse, we obtain $x_{\mathrm{LC}, k_{1}}<x_{\mathrm{LC}, k_{2}}$ [Fig. 4(b)], which indicates that the long pulse always affects the expression of $x_{j_{2}}$ whereas it influences $x_{j_{1}}$ only a little. In contrast, the short pulse affects $x_{j_{1}}$ more strongly than $x_{j_{2}}$. Our result shows that, provided the circadian clocks are designed optimally, long and short pulses affect the expression of two different components (Per2 and Per1) differently. Surprisingly, our expression patterns agree with the experiments of Schwartz et al. [15]. They hypothesized that light stimuli affect the transcription of Per 1 and the degradation of Per2. In their molecular terms, the $k_{1}$ th and $k_{2}$ th species in our framework correspond to Bmal1 and Per2, which regulate the light effect (transcription and degradation) on Per1 and Per2, respectively [i.e., $x_{k_{1}}=\left[\right.$ Bmal1] and $x_{k_{2}}=[$ Per 2$]$, Fig. 4(c)]. The phase difference between Per2 and Bmal1 was experimentally determined as $\nu \sim 2$ [23] and close to our result $(\nu=1.47)$.

The pPRCs hitherto discussed are intrinsic in the sense that they represent the internal clock dynamics. The intrinsic pPRCs can be observed only through the phase shift induced by short light pulses [14]. Theoretically, precise measurement is possible only through $\delta$-peaked stimuli. In experiments involving higher organisms, however, light pulses are much longer than the $\delta$-peaked function, and observed pPRCs become different from the intrinsic ones. To study the relation between intrinsic and observed pPRCs, let us consider a squared-pulse stimulation $d \rho=\chi p(t)$ with $p(t)=\ell^{-1} H\left(t-t_{s}\right) H\left(\ell+t_{s}-t\right)$ where $H(t)$ is the Heaviside step function, $t_{s}$ is onset time of the pulses and $\ell$ is the pulse duration. For $\ell \rightarrow 0$, the squared pulse reduces to a $\delta$ function $\delta\left(t-t_{s}\right)$. Let $\tilde{Z}(\phi ; \ell)$ be an observed pPRC of $Z(\phi)$ by a light pulse with the duration $\ell$. Observed and intrinsic pPRCs can be related via $c_{\mu}=-\mathrm{i} \tilde{c}_{\mu} \ell \mu \Omega /\{\chi[1-\exp (\mathrm{i} \mu \Omega \ell)]\}$ for $\mu \neq 0$ and $c_{\mu}=$ $\tilde{c}_{\mu} / \chi$ for $\mu=0$, where $c_{\mu}$ and $\tilde{c}_{\mu}$ are Fourier coefficients of intrinsic and observed pPRCs, respectively $[Z(\phi)=$ $\sum_{\mu=-N_{\mu}}^{N_{\mu}} c_{\mu} \exp (\mathrm{i} \mu \phi)$ and $\tilde{Z}(\phi ; \ell)=\sum_{\mu=-N_{\mu}}^{N_{\mu}} \tilde{c}_{\mu} \exp (\mathrm{i} \mu \phi)$ with $N_{\mu}$ being an expansion order]. By this method, we inferred the intrinsic pPRC from an observed pPRC in humans [24] $(\ell=6.7 \mathrm{~h})$, where a dead zone is seemingly nonexistent [19]. The inferred pPRC (dashed line) and the observed pPRC (solid line) are shown in Fig. 4(d), where the phase (horizontal axis) represents the onset of the pulse. This result suggests that superficial pPRCs may lack a dead zone even though their innate mechanisms actually do.

We have demonstrated that key properties of circadian clocks are consequences of optimization to attain the maximal limit of entrainability and regularity. Our theory explains known experimental results such as the role sharing of two inputs and different gene expression patterns by different pulses. We also explain the 
superficial absence of a dead zone in humans. The model can be used to reveal key molecular elements responsible for the clock.

This work was supported by Grant-in-Aid for Young Scientists B (Y. H., No. 25870171) and for Innovative Areas "Biosynthetic machinery" (M. A.) from Ministry of Education, Culture, Sports, Science, and Technology (MEXT), Japan.

*Present address: Department of Information and Communication Engineering, Graduate School of Information Science and Technology, The University of Tokyo, Tokyo 113-8656, Japan.

Corresponding author. yoshihiko.hasegawa@gmail.com

[1] M. W. Young and S. A. Kay, Nat. Rev. Genet. 2, 702 (2001).

[2] C. H. Johnson, P. L. Stewart, and M. Egli, Annu. Rev. Biophys. 40, 143 (2011).

[3] J. M. G. Vilar, H. Y. Kueh, N. Barkai, and S. Leibler, Proc. Natl. Acad. Sci. U.S.A. 99, 5988 (2002).

[4] D. Gonze, J. Halloy, and A. Goldbeter, Proc. Natl. Acad. Sci. U.S.A. 99, 673 (2002).

[5] E. D. Herzog, S. J. Aton, R. Numano, Y. Sakaki, and H. Tei, J. Biol. Rhythms 19, 35 (2004).

[6] D. Gonze and A. Goldbeter, J. Stat. Phys. 101, 649 (2000).

[7] T. Roenneberg, S. Daan, and M. Merrow, J. Biol. Rhythms 18, 183 (2003).

[8] D. A. Golombek and R. E. Rosenstein, Physiol. Rev. 90, 1063 (2010).
[9] Y. Hasegawa and M. Arita, J. R. Soc. Interface 11, 20131018 (2014).

[10] C. Troein, J. C. Locke, M. S. Turner, and A. J. Millar, Curr. Biol. 19, 1961 (2009).

[11] C. Troein, F. Corellou, L. E. Dixon, G. van Ooijen, J. S. O'Neill, F.-Y. Bouget, and A. J. Millar, Plant J. 66, 375 (2011).

[12] U. Albrecht, B. Zheng, D. Larkin, Z. S. Sun, and C. C. Lee, J. Biol. Rhythms 16, 100 (2001).

[13] S. Steinlechner, B. Jacobmeier, F. Scherbarth, H. Dernbach, F. Kruse, and U. Albrecht, J. Biol. Rhythms 17, 202 (2002).

[14] R. Refinetti, Circadian Physiology, 2nd ed. (Taylor \& Francis, London, 2005).

[15] W. J. Schwartz, M. Tavakoli-Nezhad, C. M. Lambert, D. R. Weaver, and H. O. de la Iglesia, Proc. Natl. Acad. Sci. U.S.A. 108, 17219 (2011).

[16] Y. Kuramoto, Chemical Oscillations, Waves, and Turbulence (Dover, Mineola, NY, 2003).

[17] S. R. Taylor, R. Gunawan, L. R. Petzold, and F. J. Doyle III, IEEE Trans. Autom. Control 53, 177 (2008).

[18] Y. Hasegawa and M. Arita, J. R. Soc. Interface 10, 20121020 (2013).

[19] See Supplemental Material at http://link.aps.org/ supplemental/10.1103/PhysRevLett.113.108101 for detailed derivations of equations, which includes Ref. [20].

[20] R. Storn and K. Price, J. Global Optim. 11, 341 (1997).

[21] A. Johnsson and W. Engelmann, in Photobiology: The Science of Life and Light (Springer, New York, 2007).

[22] D. L. Hartmann, Global Physical Climatology (Academic Press, New York, 1994).

[23] H. R. Ueda et al., Nature (London) 418, 534 (2002).

[24] S. B. S. Khalsa, M. E. Jewett, C. Cajochen, and C. A. Czeisler, J. Physiol. 549, 945 (2003). 